Bangladesh J. Bot. 49(4): 1029-1035, 2020 (December)

\title{
ANTIOXIDANT PHENOLIC COMPOUNDS FROM CHINESE WHITE OLIVE (CANARIUM ALBUM L.)
}

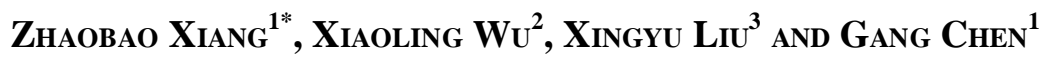 \\ College of Environment and Resources, Chongqing Technology \\ and Business University, Chongqing-400067, China
}

Keywords: Chinese white olive, Phenolic compounds, Chromatography separation

\begin{abstract}
To find antioxidant compounds from Chinese white olive (CWO) based on bioassay-guided method, the ethanol extract and corresponding 4 fractions were evaluated for their antioxidant activities by DPPH and ABTS assay. The results revealed that the AcOEt fraction had the most potent antioxidant activity. Further, six compounds isolated from the AcOEt fraction, and their structure were identified as (-)-epicatechin gallate (i), ellagic acid (ii), gallic acid (iii), (-)-epigallocatechin (iv), (-)-epigallocatechin gallate (v) and kaempferol (VI) by MS and NMR spectrum. Compounds I, IV and V were isolated and identified from CWO for the first time. All the compounds (I-VI) exhibited potent antioxidant activity. Among them, compound III showed best antioxidant activity in both DPPH and $\mathrm{ABTS}^{+}$assay. The phenolic compounds from CWO with potent antioxidant activities were responsible for the antioxidant activity of CWO extract.
\end{abstract}

\section{Introduction}

Chinese white olive (CWO, Canarium album L.), usually called Ganlan or Qingguo in China, is a well-known Chinese fruit tree belonging to the Burseraceae. It is indigenous to the southeast area of China, and then has been introduced to other Asian tropical and semi-tropical regions (Xiang et al. 2014). The fresh fruit of CWO is light colored and edible, with low oil and rich nutrition. Most fruits are generally processed in food industry to beverage, candy and confections. The dried fruit is also used as traditional medicine for treatment of faucitis, stomatitis, hepatitis, and toxicosis in China (Xiang and $\mathrm{Wu}$ 2017). According to modern pharmacological investigations, CWO fruits possess some pharmacological properties such as antibacterial (Xiang et al. 2013), anti-alcoholic and hepatoprotective (Zhu et al. 2010), anti-HIV activities (Duan et al. 2013) and antiglycation (Kuo et al. 2015). However, the fundamental research of CWO is rather poor. Particularly, there was no report on discovery of active compounds based on activity tracking from CWO.

Oxidative modification of DNA, proteins, lipid and small cellular molecules by reactive oxygen species (ROS) increases the risk of a wide range of common degenerative diseases such as osteoporosis, cancer and cardiovascular diseases. Antioxidants are of great importance in terms of reducing ROS so as to lower the incidence rate of degenerative diseases. Increasing dietary intake of natural antioxidants, such as polyphenolic compounds and antioxidant vitamins, has been shown to have an inverse association with the risks of cardiovascular diseases, inflammatory conditions, neurodegenerative diseases, diabetes mellitus and cancer (Xiang and Wu 2017). Phytochemical investigations revealed that phenolic compounds have been regarded as representative components of the plant. However, there was no report on discovery of antioxidant

*Author for correspondence: <xiangzb@126.com>. ${ }^{1}$ Key Laboratory of Natural Medicine Research of Chongqing Education Commission, Chongqing Technology and Business University, Chongqing-400067, China. ${ }^{2}$ Academic Quality Inspection and Evaluation Office, Chongqing Technology and Business University, Chongqing 400067, China. ${ }^{3}$ College of Bioengineering, Chongqing University, Chongqing400044, China. 
phenolic compounds based on activity tracking. Therefore, the aim of this study was to find antioxidant phenolic compounds based on bioassay-guided method from CWO using DPPH. and $\mathrm{ABTS}^{+}$. assay and separation by chromatography.

\section{Materials and Methods}

Ethanol, PE $\left(60-90^{\circ} \mathrm{C}\right)$, AcOEt, n-butanol, methanol, chloroform $\left(\mathrm{CHCl}_{3}\right)$ (all analytical grade) were purchased from Chongqing East Chemical Industry Co., Ltd. (Chongqing, China). Sephadex LH-20 was the product of GE Healthcare Bio-Sciences AB (Uppsala, Sweden). 1,1diphenyl-2-picrylhydrazyl (DPPH) and 2,2'-azinobis-(3-ethylbenzothiazoline-6-sulfonate) (AB TS) were purchased from Sigma Chemical Co. (Missouri, USA). Silica gel (200 - 300 mesh) for column chromatography and silica gel $\mathrm{GF}_{254}$ for TLC were obtained from Qingdao Marine Chemical Company (Qingdao, China).The plant materials were collected in September, 2016 from Jiangjin, Chongqing municipality and identified as the dried fruits of Canarium album (Lour.) Raeusch by Professor Ren S.G., College of Bioengineering, Chongqing University, China.

The ethanol extract from fruits of CWO was obtained by heating at $70^{\circ} \mathrm{C}$ for $8 \mathrm{hrs}$ in a water bath (H-6, Ronghua, Jiangsu, China) using $80 \%$ ethanol as solvent after soaking 15 days, After removal of solvent under vacuum with rotary evaporator( RE-5205,Yarong, Shanghai, China), most of the residue was dispersed by water and extracted with PE $(4 \times 2 \mathrm{~L}$, each $24 \mathrm{hrs})$, AcOEt (4 $\times 2 \mathrm{~L}$, each $24 \mathrm{hrs})$ and $n$-butanol $(4 \times 2 \mathrm{~L}$, each $24 \mathrm{hrs})$ successively to obtain 4 fractions, namely PE, AcOEt, $n$-butanol and water fraction. The DPPH and $\mathrm{ABTS}^{+}$assay was performed according to literature (Xiang et al. 2017) and Vitamin C (Vc) as positive control group.

The EtOAc fraction was dissolved in EtOAc and extracted with $5 \%$ sodium bicarbonate aqueous solution, $5 \%$ sodium carbonate aqueous solution and $2 \%$ sodium hydroxide aqueous solution successively to give four subfractions $B_{1}-B_{4}$. Subfraction $B_{1}$ was purified over silica gel with $\mathrm{CHCl}_{3}-\mathrm{MeOH}$ to obtain four mixtures $\mathrm{M}_{1}-\mathrm{M}_{4}$. Mixture $\mathrm{M}_{1}$ was purified over silica gel (Qingdao Marine, Shandong, China) with PE- acetone repeatedly to afford compounds I and II. Mixture $\mathrm{M}_{3}$ was purified over silica gel with $\mathrm{CHCl}_{3}-\mathrm{MeOH}$ repeatedly to afford compounds III and IV. Subfraction $\mathrm{B}_{2}$ was purified over silica gel with $\mathrm{CHCl}_{3}-\mathrm{MeOH}$ to obtain three mixtures $\mathrm{M}_{5}-\mathrm{M}_{7}$. Mixture $\mathrm{M}_{6}$ was purified over silica gel with $\mathrm{CHCl}_{3}-\mathrm{MeOH}$ and Sephadex LH-20 (GE, Uppsala, Sweden) repeatedly to afford compound V. Mixture $\mathrm{M}_{7}$ was purified over silica gel with $\mathrm{CHCl}_{3}-\mathrm{MeOH}$ and sephadex LH-20 repeatedly to afford compound VI. The NMR data were recorded on Bruker spectrometers (AV-500, Germany) using DMSO- $\mathrm{d}_{6}$ as solvent and TMS as internal reference. ESI-MS and HR-ESI-MS were performed with Mat-212 and Micromass Auto Spec Q-TOF spectrometers (Micromass, U.K.), respectively.

\section{Results and Discussion}

The antioxidant activity of the extracts of CWO was reported by Ho and Luo (2015). However, there was no report on discovery of antioxidant fraction and compounds based on bioassay-guided method from CWO. There are so many methods to evaluate the potential antioxidant activity due to different mechanisms. DPPH. and $\mathrm{ABTS}^{+}$assays are both especially effective way to test the antioxidant activity. In the DPPH. assay, there existed a good linear correlation between antioxidant activities and concentrations of ethanol extract and four fractions of $\mathrm{CWO}$ in certain range of concentrations, while, they arose a high concentrations, the antioxidant activities tended to vary gently (Fig. 1). The $\mathrm{SC}_{50}$ value as follow: $0.038 \mathrm{mg} / \mathrm{ml}$ for $\mathrm{Vc}$ used as positive control and $4.429 \mathrm{mg} / \mathrm{ml}$ for ethanol extract. It is obvious that the ethanol extract of CWO expressed good DPPH radical scavenging activity. Among the four fractions, the AcOEt exhibited the best DPPH scavenging activity with the minimal $\mathrm{SC}_{50}$ value $(1.654 \mathrm{mg} / \mathrm{ml})$, 
meanwhile, the water fraction exhibited the worst activity with the maximal $\mathrm{SC}_{50}$ value $(8.826$ $\mathrm{mg} / \mathrm{ml}$ ). The PE fraction owned some radical scavenging activity in DPPH. system with the good $\mathrm{SC}_{50}$ value $(4.484 \mathrm{mg} / \mathrm{ml})$, though it is weaker than n-butanol fraction with the good $\mathrm{SC}_{50}$ value (3.908 $\mathrm{mg} / \mathrm{ml})$.

In order to evaluate antioxidant activity more credibly, $\mathrm{ABTS}^{+}$system for determination of total antioxidant capacity was adopted. In the $\mathrm{ABTS}^{+}$assay, there existed a good linear correlation between antioxidant activities and concentrations of ethanol extract and four fractions in certain range of concentrations. Based on the $\mathrm{ABTS}^{+}$scavenging activities in Fig. 2. It is obvious that the ethanol extract of CWO expressed good $\mathrm{ABTS}^{+}$radical scavenging activity, though it is weaker than Vc. Among the four fractions, the AcOEt exhibited the best $\mathrm{ABTS}^{+}$scavenging activity with the minimal $\mathrm{SC}_{50}$ value $(0.477 \mathrm{mg} / \mathrm{ml})$, meanwhile, the water fraction exhibited the worst activity.

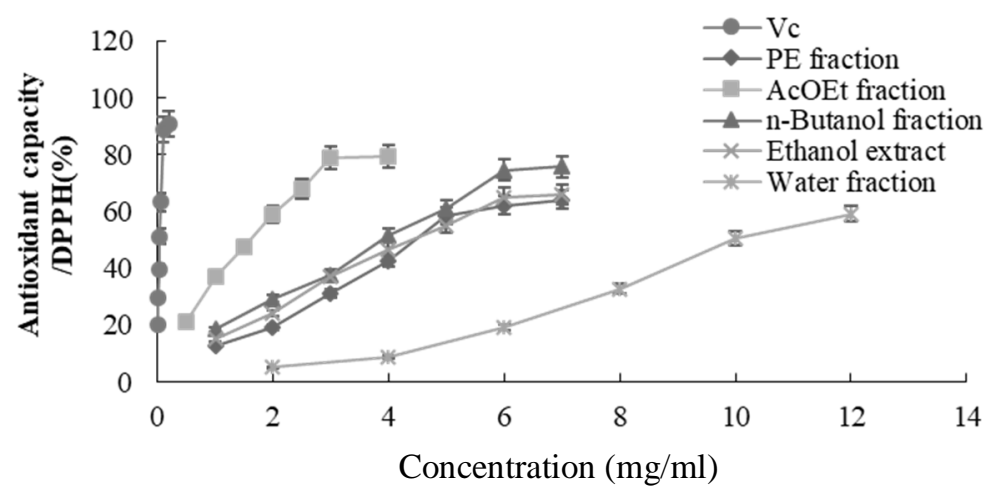

Fig. 1. DPPH. scavenging activities of ethanol extract and 4 fractions of CWO.

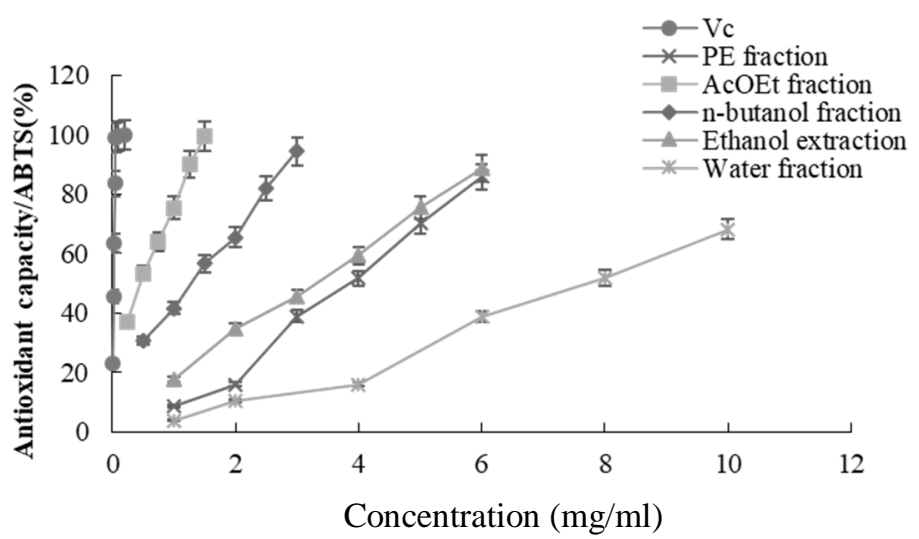

Fig. 2. $\mathrm{ABTS}^{+}$scavenging activities of ethanol extract and 4 fractions of CWO.

The role of free radicals and active oxygen is becoming increasingly recognized in the pathogenesis of the many human diseases, including cellular damage, cancer, aging and atherosclerosis (Zheng et al. 2013). Although oxidation could be prevented in foods by the addition of synthetic antioxidants such as BHT, BHA and TBHQ, but more attention has recently been paid to natural antioxidants because of potential toxicities and carcinogenic effects of these synthetic antioxidants (Wang 2014). The antioxidant activity of CWO was reported, but most of them focused on the activity owning main compositions, such as tannins, total polyphenols (Zhang 
et al. 2008), total polysaccharide (Zhu et al. 2013), and so on. In fact, the tannins, total polyphenols and total polysaccharide obtained from CWO were impure and even low in content. Therefore, antioxidant activity may also come from other components. It is very important to separate the extract systematically for seeking the antioxidant compound. Among the four fractions, the AcOEt fraction exhibited the best antioxidant activity with the minimal $\mathrm{SC}_{50}$ value, so it is the most likely to find antioxidant compound from the fraction.

Compound I, a white powder with good solubility in ethanol and ethyl acetate, $\mathrm{FeCl}_{3}$ reaction was positive. It exhibited a quasi-molecularion ion peak at $\mathrm{m} / z 443[\mathrm{M}+\mathrm{H}]^{+}$by ESI-MS and the molecular formula $\mathrm{C}_{22} \mathrm{H}_{18} \mathrm{O}_{10}$ (Fig. 3) was determined by HRESI-MS $\left(\mathrm{m} / 2443.0833[\mathrm{M}+\mathrm{H}]^{+}\right)$. All the NMR spectral data were consistent with Fu et al. (2012), and compound I was identified as (-)epicatechin gallate and first found from CWO.

Compound II, a light-yellow powder with good solubility in ethanol and ethyl acetate, $\mathrm{FeCl}_{3}$ reaction was positive. It exhibited a quasi-molecularion ion peak at $\mathrm{m} / z 301[\mathrm{M}-\mathrm{H}]^{-}$by ESI-MS and the molecular formula $\mathrm{C}_{14} \mathrm{H}_{6} \mathrm{O}_{8}$ was determined by HRESI-MS $\left(\mathrm{m} / 2300.9985[\mathrm{M}-\mathrm{H}]^{-}\right)$. All the NMR spectral data were consistent with those reported by Shi et al. (2006), and compound II was identified as ellagic acid.

Compound III, a white powder with good solubility in ethanol and ethyl acetate, $\mathrm{FeCl}_{3}$ reaction was positive. It exhibited a quasi-molecularion ion peak at $\mathrm{m} / z 169[\mathrm{M}-\mathrm{H}]^{-}$by ESI-MS and the molecular formula $\mathrm{C}_{7} \mathrm{H}_{6} \mathrm{O}_{5}$ was determined by HRESI-MS $\left(\mathrm{m} / z\right.$ 169.0145 [M-H] $\left.{ }^{-}\right)$. All the NMR spectral data were consistent with those reported by Shi et al. (2006), and compound III was identified as gallic acid.
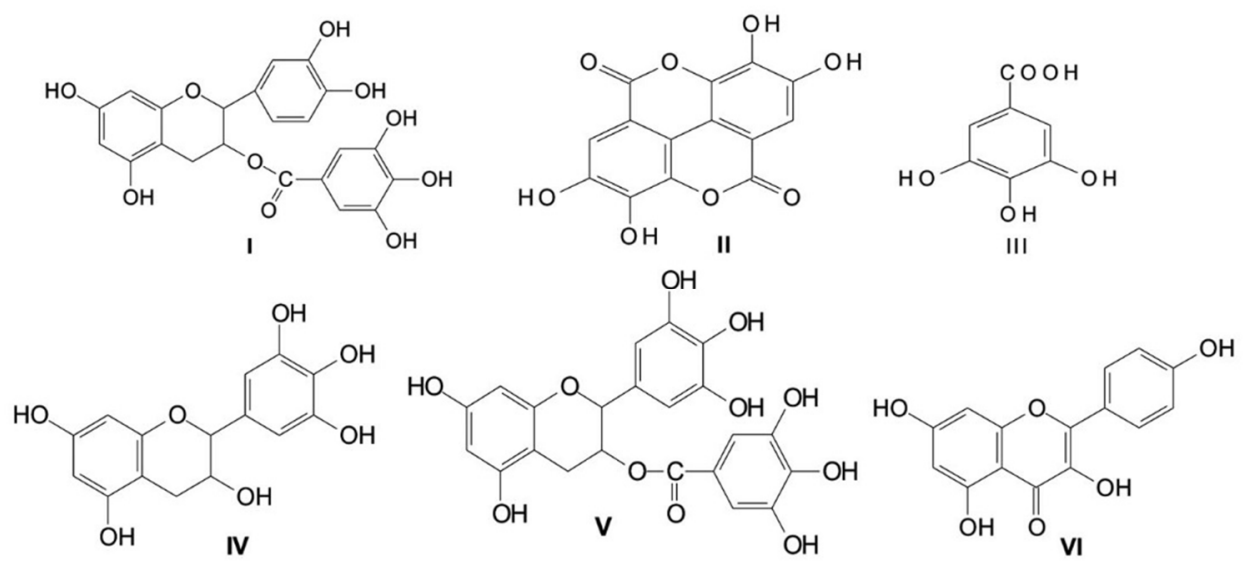

Fig. 3. Chemical compositions from antioxidant fraction of CWO.

Compound IV, a white powder with good solubility in ethanol and water, $\mathrm{FeCl}_{3}$ reaction was positive. It exhibited a quasi-molecularion ion peak at $\mathrm{m} / z 307[\mathrm{M}+\mathrm{H}]^{+}$by ESI-MS and the molecular formula $\mathrm{C}_{15} \mathrm{H}_{14} \mathrm{O}_{7}$ was determined by HRESI-MS $\left(\mathrm{m} / z\right.$ z $\left.307.0717[\mathrm{M}+\mathrm{H}]^{+}\right)$. All the NMR spectral data were consistent with those reported by Jia et al. (1998) and compound IV was identified as (-)-epigallocatechin and first found from CWO.

Compound $\mathbf{V}$, a white powder, $\mathrm{FeCl}_{3}$ reaction was positive. It exhibited a quasi-molecularion ion peak at $m / z 459[\mathrm{M}+\mathrm{H}]^{+}$by ESI-MS and the molecular formula $\mathrm{C}_{22} \mathrm{H}_{18} \mathrm{O}_{11}$ was determined by HRESI-MS $\left(\mathrm{m} / z 459.3373[\mathrm{M}+\mathrm{H}]^{+}\right)$. All the NMR spectral data were consistent with Zhao et al. (2012), and compound V was identified as (-)-epigallocatechin gallate and first found from CWO. 
Compound VI, a yellow powder, the color of $\mathrm{FeCl}_{3}$ reaction was yellow green. It exhibited a quasi-molecularion ion peak at $\mathrm{m} / z 287[\mathrm{M}+\mathrm{H}]^{+}$by ESI-MS and the molecular formula $\mathrm{C}_{15} \mathrm{H}_{10} \mathrm{O}_{6}$ was determined by HRESI- MS $\left(\mathrm{m} / z 287.0468[\mathrm{M}+\mathrm{H}]^{+}\right)$. All the NMR spectral data were consistent with those reported by $\mathrm{Hu}$ et al. (2003), and compound VI was identified as kaempferol.

In the DPPH. assay, there existed a good linear correlation between antioxidant activities and concentrations of all the compounds (I-VI) from antioxidant fraction of CWO in certain range of concentrations, while they arose a high concentration, the antioxidant activities tended to vary gently. The $\mathrm{SC}_{50}$ value as follow: $0.038 \mathrm{mg} / \mathrm{ml}$ for $\mathrm{Vc}$ used as positive control, $0.194 \mathrm{mg} / \mathrm{ml}$ for compound I, $0.055 \mathrm{mg} / \mathrm{ml}$ for compound II, $0.026 \mathrm{mg} / \mathrm{ml}$ for compound III, $0.102 \mathrm{mg} / \mathrm{ml}$ for compound IV, $0.060 \mathrm{mg} / \mathrm{ml}$ for compound V and $0.206 \mathrm{mg} / \mathrm{ml}$ for compound VI. Based on the DPPH scavenging activities in Fig. 4. It is obvious that all the compounds (I-VI) from antioxidant fraction of CWO expressed potent DPPH. radical scavenging activity, especially the compound III is better than Vc used as positive control widely. Furthermore, there are two compounds (II and V) exhibiting similar DPPH. scavenging activity with $\mathrm{Vc}$. Even, the $\mathrm{SC}_{50}$ value of compound VI with the worst activity is only $0.206 \mathrm{mg} / \mathrm{ml}$. So, all the compounds (I-VI) showed good radical scavenging activity in DPPH. system.

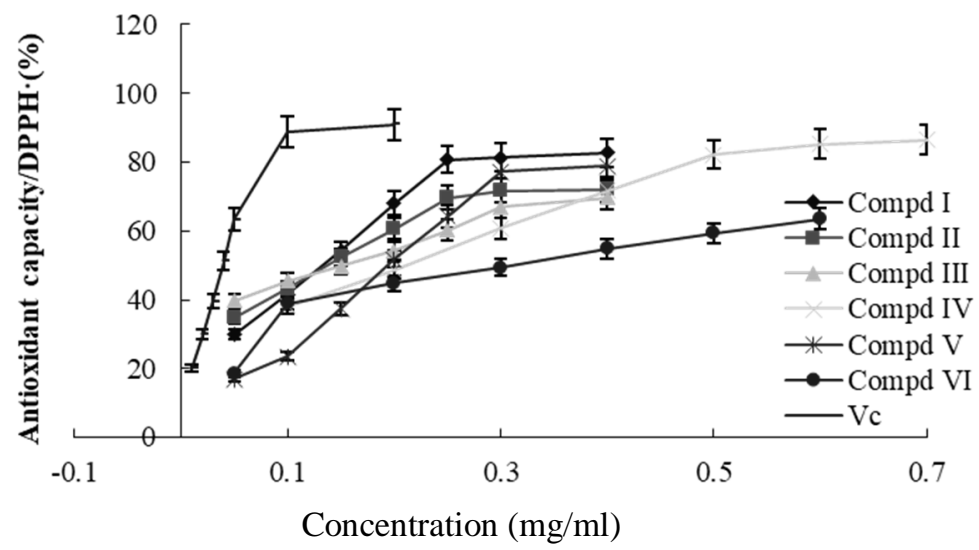

Fig. 4. DPPH scavenging activities of compounds I-VI from antioxidant fraction of CWO.

In order to evaluate antioxidant activity of all the compounds (I-VI) more credibly, $\mathrm{ABTS}^{+}$ system for determination of antioxidant capacity also has been adopted. In the $\mathrm{ABTS}^{+}$assay, there existed a good linear correlation between antioxidant activities and concentrations of all the compounds (I-VI) in certain range of concentrations similarly. Based on the $\mathrm{ABTS}^{+}$scavenging activities in Fig. 5. It is obvious that all the compounds (I-VI) from antioxidant fraction expressed potent $\mathrm{ABTS}^{+}$radical scavenging activity with the very good $\mathrm{SC}_{50}$ value $(0.023-0.040 \mathrm{mg} / \mathrm{ml})$, which is in accordance with the previous DPPH assay, similarly the $\mathrm{ABTS}^{+}$scavenging activity of compound III is better than that of $\mathrm{Vc}$ according to the $\mathrm{SC}_{50}$ value. The former is $0.023 \mathrm{mg} / \mathrm{ml}$, and the latter is $0.028 \mathrm{mg} / \mathrm{ml}$.

All the compounds (I-VI) exhibited very good antioxidant activity in the present study, especially the antioxidant activity of compound III is better than that of $\mathrm{Vc}$ used as positive control widely. The results were consistent with that phenolic compounds including flavones are well-known antioxidants (Peng et al. 2018). Phenolic compounds are secondary metabolites widely found in fruits, mostly represented by flavonoids and phenolic acids. Studies have shown 


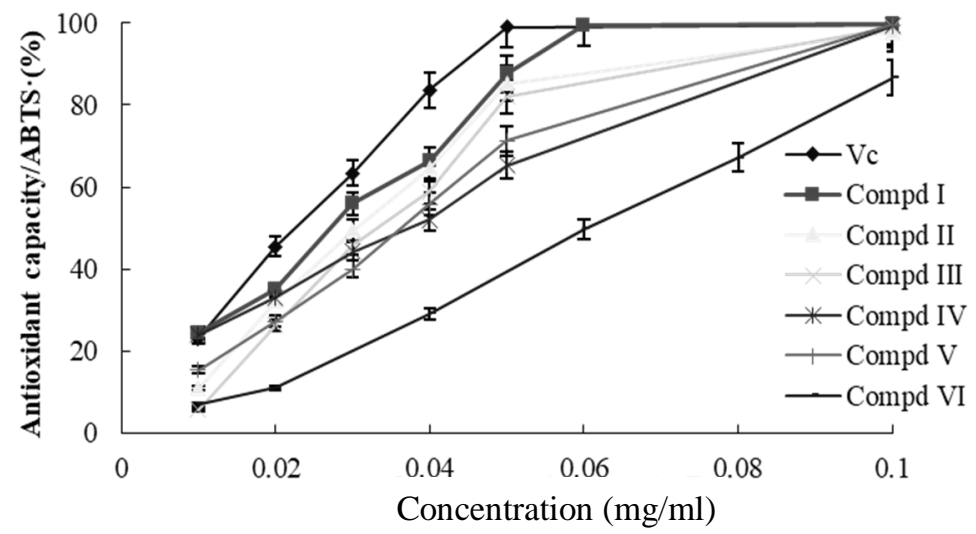

Fig. 5. $\mathrm{ABTS}^{+}$scavenging activities of compounds I-VI from antioxidant fraction of CWO.

the importance of the regular consumption of fruits, especially for preventing diseases associated with oxidative stress. From the present result it may concluded that all the compounds (I-VI), especially compound III obtained from the CWO fruit could be used for good natural antioxidants. Phenolic compounds from CWO with potent antioxidant activities were responsible for the antioxidant activity of CWO extract.

\section{Acknowledgments}

This work was financially supported by the Key Laboratory of Natural Medicine Research of Chongqing Education Commission (KFJJ2019094) and Scientific Research Foundation of Chongqing Technology and Business University (2015-56-07).

\section{References}

Duan WJ, Tan SY, Chen J, Liu SW, Jiang SB, Xiang HY and Xie Y 2013.Isolation of anti-HIV components from Canarium album fruits by high-speed counter-current chromatography. Anal. Lett. 46: 1057-1068.

Fu DH, Liu ZH, Huang JA, Chen JH, Cai ZA, and Zhao SJ 2012. Study on fat-reducing functional components of Fuzhuan tea. Tea Sci. 32(3): 217-223.

Ho RK and Luo HJ 2015. Antioxidant activity of olive fruit extracts and its role in skin health. Food Res. Develop. 36(6): 45-49.

Hu XL, Zhu H, Liu CR and Tu PF 2003. Study on chemical composition of impatiens. Chin. Medi. 25(10): 833-834.

Jia ZS, Zhou B, Yang L, Wu LM and Liu ZL 1998. 2D NMR study of tea polyphenols in green tea. Pop Mag. 15(1): 23-30.

Kuo CT, Liu TH and Hsu TH 2015. Antioxidant and antiglycation properties of different solvent extracts from Chinese olive (Canarium album L.) fruit. Asian Pacific J. Tropi. Medi. 16(3): 156-158.

Peng MY, Liu JY, Liu ZJ, Fu B and Xu N 2018. Effect of citrus peel on phenolic compounds, organic acids and antioxidant activity of soy sauce. LWT. 90: 627-635.

Shi XH, Du XL and Kong LY 2006. Study on the chemical composition of roots of Junggar. Chin. J. Tradi. Chin. Med. 31(18): 1503-1506.

Wang XH 2014. Study on the antioxidative activity of three natural antioxidants in the healthy mix pine oils. J. Chongqing Technol. Business Univ. (Nat. Sci. Ed.) 31(2): 95-100. 
Xiang ZB, Hu B and He CL 2013.Study on antibacterial activity of different fractions from Canarium album. Sci. Technol. Food Industry 34(12): 149-152.

Xiang ZB, Liu XY, He CL and Heng LS 2014. Flavonoids from Canarium album. Asian J. Chem. 14: 45294530.

Xiang ZB and Wu XL 2017. Chemical constituents of Chinese white olive. Pharm. Chem. J. 51: 465-470.

Xiang ZB, Wu XL and Liu XY 2017. Chemical compisition and antioxidant activity of petroleum ether extract of Canarium album. Pharm. Chem. J. 51(7): 606-611.

Zhao ZX, Jin J, Lin ZZ, Zhu CX, Zeng RX and Fan Z 2012. Study on chemical composition of ethyl acetatein cane and cane. Modern Medi. Clinical. 27(3): 200-203.

Zhang LL, Yang ZW and Lin YM 2008. Study on antioxidant activity of polyphenols from Canarium album fruits. Sci. Technol. Food Industry 29(4): 57-59.

Zheng MC, Fan XM and Guang HZ 2013. Optimization of extraction process of olive leaves by RSM and evaluation of antioxidant capacity measurements to the mixtures. J. Food Sci. Eng. 10(3): 525-533.

Zhu L, Zhao GX, Shen YW and Chen Z 2010. Protection effect of flavone of Canarium album (lour.) against alcohol-induced liver injury in mice. Food Machinery 26(3): 91-93.

Zhu JF, Deng YX, Ran YL, Tang CH and Yu HY 2012. Antioxidant activities of perilla meal polysaccharide PWPS. J. Chongqing Technol. Business Univ. (Nat. Sci. Ed.) 29(12): 83-86.

(Manuscript received on 29 July, 2019; revised on 28 March, 2020) 